\title{
Resource Control and Visionary Leadership: Two Exercises
}

\author{
Cindy P. Lindsay, Indiana University
}

Cathy A. Enz, Cornell University

The two exercises presented here use five sets of Tinkertoys to illustrate complex management issues. In the first exercise, "Tinkertoy Power," participants explore issues of resource dependence, power, and ethics. The second exercise, "Blind Leadership," focuses on the importance of leaders communicating a vision to subordinates.

\section{Tinkertoy Power}

Group Size

Four groups of 3 to 10 members each.

Materials Needed

Five sets of "basic" Tinkertoys.

Time Required

Approximately 1 hour.

Objective of the Exercise

"Tinkertoy Power" is a bargaining and resource control exercise that involves participants in a complex set of group power relations. For the exercise, four groups are created. Each group views a central model of a structure to be built with a set of Tinkertoys (see Figure 1). Each group is awarded points for completing its structure. Groups may also accumulate points by having valued excess resources. Excess resources only provide a team with points after its structure is completed. If the structure is not complete, no points are assigned.

The objective of getting the most points may be explicitly stated and rewarded or not, depending on the wishes of the instructor. If the objective is not specified, the instructor can later discuss the implicit assumptions and social definitions team members attached to the "game." 


\section{Point Assignment}

Completed structure

Excess Resources:

Blue and Yellow sticks

White connectors

Red and Green sticks
500 points

10 points each

5 points each

1 point each

\section{Resource-Based Group Composition}

Group 1. This group is given nearly all of the pieces it needs to complete the structure and is thus in control of most of the necessary resources. This group also possesses many of the pieces needed by other groups to complete their structures. Therefore, this group is, the resources-rich and powerful group.

Group 2. Group 2 has the most Tinkertoys of any group but few of the pieces that it or the other groups need to successfully build the structure. Although at first it may appear to be resource-rich, this group is resource-poor and powerless.

Groups 3 and 4. These groups are provided with a moderate amount of the pieces that they need to build their structures. Each of these groups must rely to some extent on other groups for critical resources, but neither group is as resource-poor as is Group 2.

Distribution of Tinkertoys to Groups

Group 1 - Resource Rich 22 red sticks 9 green sticks

11 blue sticks 14 yellow sticks 18 wooden spools 9 red wheels

8 white connectors

9 long orange connectors

4 short orange connectors

2 people

1 flag

Group 2 - Resource Poor

6 red sticks

1 green stick

25 blue sticks 
38 yellow sticks

18 wooden spools

3 red wheels

32 white connectors

2 long orange connectors

2 short orange connectors

0 people

11 flags

Groups 3 and 4 - Middle Class Groups

10 red sticks

3 green sticks

6 blue sticks

6 yellow sticks

10 wooden spools

2 red wheels

16 white connectors

2 long orange connectors

5 short orange connectors

1 person

2 flags 


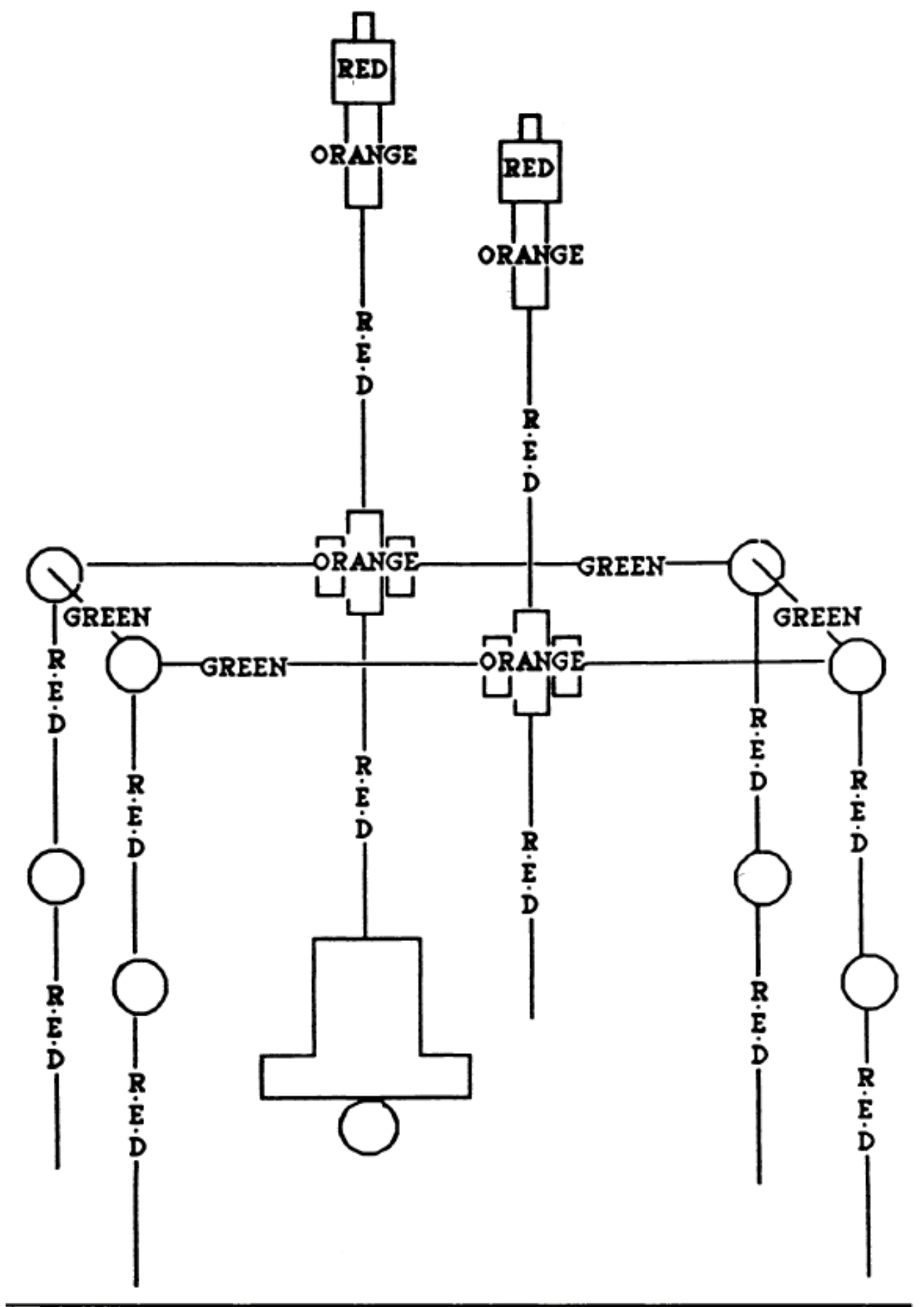

Figure 1: Model Structure for Tinkertoy Power

Instructions to Participants

Basic instructions include a description of the structure and the point values for the completed structure and excess resources. Additional instructions to participants may be given by the instructor at the instructor's discretion. Such added instructions may include the following: 
1. You may negotiate/bargain across groups for resources.

2. You are competing for the most points.

3. You have 20 minutes before point totals are counted.

4. There are no rules other than those explicitly stated.

5. The first 5 minutes of the exercise may only be used to plan your strategy. You may not bargain or put pieces together until after this initial 5-minute planning period is over.

\section{Factors to Consider When Running the Exercise}

Often, participants take pieces off the model structure to use in their own structure. This can be used as a basis for discussion of common resources and ethics. For example, the instructor could ask, " $f s$ taking pieces off the model unethical or innovative, and what makes the difference?"

It has also been our experience that participants ask the instructor for permission to do various acts (such as creating coalitions, conducting unequal trades, or removal of pieces from the model). The instructor may wish to act as authority figure or remain neutral. Different instructor choices may lead to discussion of ethics, innovation, and authority. We prefer being neutral, thus forcing the answer to the question of ethical choice to be made at the student level.

In addition, the basic exercise may be altered to fit class or instructor needs. For example, money or other incentives may be tied to point totals if the instructor wants to emphasize competition or to create more investment by the participants in the exercise. Alternatively, group leaders may be assigned to each group if leadership skills are to be addressed in class. Finally, delegating responsibility and subdividing tasks can be suggested to stimulate organizational design or intragroup authority issues. Issues for Discussion after the Exercise

Issues for discussion after the exercise may include

1. resource allocation and resource dependence

2. power and influence strategies

3. coalition, cooptation, bargaining

4. rules, who makes them and how - implicit rules/norms or legitimate power

5. resource allocation and rules

6. win-win versus win-lose assumptions - across and within groups

7. ethics, morals, rules, and innovation

8. leadership and interpersonal styles

9. group cohesion and competition 
10. participative, organic, or mechanistic structure of roles and responsibilities.

In the exercise, the unequal allocations of resources create power differences between groups. As a result, the groups may develop different strategies for obtaining the needed resources. Some groups bargain for primary resources needed in the construction of the structure by trading secondary resources (those with value only after construction) to groups rich in primary resources. Others may try to form a coalition with other relatively resource- poor groups. In general, bargaining is attempted first. Sometimes bargaining attempts do not succeed because the groups are too self-interested. If bargaining does not succeed, or if negative interactions occur, coalition formation is a likely second strategy. Unfavorable bargains or negative responses on the part of the powerful group make the formation of coalitions among powerless groups more likely. With a common enemy, there is increased definition of ins and outs (powerful and powerless) and added incentive to trust among powerless groups. Without such incentive, coalitions may be more difficult to form and a basis for cooperation may need to be more strenuously negotiated. The use of coalition strategies is also affected by the assumptions that students bring to the exercises. Those with win-win assumptions are much more likely to jump to coalition formation as a first strategy than are those who assume competition.

Individual strategies may also be observed and discussed in the attempts to bargain and form coalitions. Reasoning, ingratiation, and assertion strategies are among the most common. In addition to these, some students may attempt to call on the higher authority of rules or on the teacher to arbitrate disputes or introduce structure.

Appeal to the rules or the teacher is especially evident if other groups try to secure resources by taking pieces from the model structure. This situation provides an excellent opportunity to discuss several aspects relating to rules and ethics. First, the legitimate power of the teacher to decide can be examined. Why is the teacher assumed to have this power? Furthermore, what if the teacher decides not to exercise this power? How are the bounds of ethical behavior and the rules decided? Is the taking of model parts creative or cheating? Students find it quite challenging and interesting to discuss their own assumptions about ethical behavior and how such behavior is generally regulated (or not) in organizations and society. This can lead to questions about what to do in situations where one feels that unethical behavior is occurring but no legitimate authority is willing to take the initiative to stop the behavior.

Intergroup dynamics and group structure can also be observed and discussed. Leadership and leadership styles, division of labor, and cohesion may all emerge. These can be discussion in relationship to assumptions about the nature of the exercise (competitive or cooperative) and the experience and 
personality of individuals involved. Group structure frequently dissolves when a group is powerless and individuals defect to other groups or attempt to pursue individual versus group goals. In contrast, powerful groups may become more cohesive and structured when group success is shared.

\section{Blind Leadership-Creating Vision}

"Blind Leadership" emphasizes the importance of leadership vision in the successful accomplishment of group tasks. In this exercise, a sighted group leader instructs blindfolded subordinates in putting together a Tinkertoy structure.

Group Size

Five groups of 4-6 people each.

Materials Needed Five sets of "basic" Tinkertoys.

One blindfold for each participant (except the leaders).

Five pictures of the Tinkertoy structure.

Time Required

Approximately 45 minutes to 1 hour.

\section{Conducting the Exercise}

Participants may self-select or be divided by the instructor into five groups. Each group is instructed to select a leader for the exercise. All subordinates are then asked to put on blindfolds. Once all subordinates are blindfolded, a picture of the structure to be built by the groups (see Figure 2 ) is handed to the leader of each group. Group leaders are told that they may do anything they wish to get their subordinates to build the structure. The only things group leaders may not do is remove the blindfolds and touch the Tinkertoys.

Issues for Discussion

After each group finishes building the structure, subordinates are asked to take off their blindfolds and give the group leader feedback. Specifically, we have them address 4 questions: (a) Does the structure look like you expected it to look; (b) what did the leader do that was helpful; (c) what did the leader do that was not helpful; (d) how did you feel about the exercise?

After each group of subordinates has given this feedback to its leader, the instructor conducts a process discussion with the entire class. 


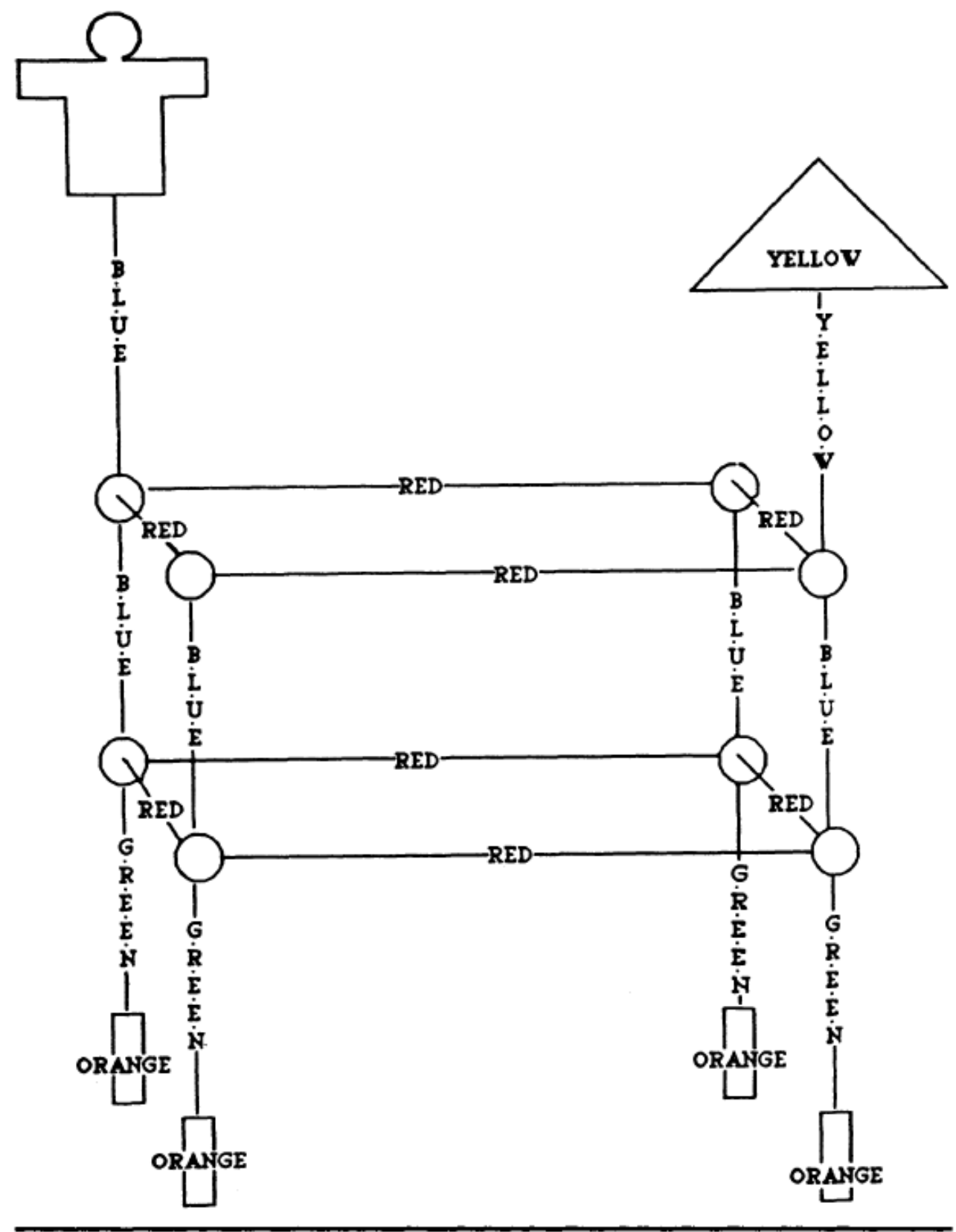

Figure 2: Structure for Blind Leadership Exercise

In our experience, is it generally true that groups that finish first and have the most accurate structures are ones in which the leader created a vision for the subordinates. That is, the most successful leaders give their subordinates a mental picture of the structure (i.e., "a box on stilts" or "a square with legs underneath and a flag pole on top"). Speed and accuracy of construction are also affected by other factors relevant to enabling subordinates to "enact the vision." Division of labor and inclusion of subordinates, use of praise and positive reinforcement by the leader, and degree of twoway communication between leader and subordinates often come up in this discussion as important 
enabling factors that may facilitate involvement but that also speed or slow construction. It is important to note that some factors that increase satisfaction with the task and enable subordinates to enact the vision may also slow construction. For example, praising the group, building team spirit, and including everyone in the construction process at every step, are all time-consuming activities that generally make the group members feel good but that may hamper the efficiency of the group. A balance of time on such activities may need to be developed; enough to create goodwill and cooperation without severely slowing construction. The discussion may be summed up by noting that successful leaders provide a vision for subordinates and then create an atmosphere in which subordinates may effectively enact the vision. Still, the effective leader must balance the tradeoffs between vision and excitement versus control and efficiency.

\section{Conclusions}

It is our experience, with both student and executive groups, that participants really enjoy the exercises. It is easy for them to analyze their own leadership behaviors and group interactions. Several students have reported that they thought the message of giving vision rather than just giving orders was so relevant that they went to their workplaces and ran the exercise with their work groups.

In summary, we have found these exercises to be simple to administer and engaging to process. The strength of both exercises is that they have a complex set of issues and outcomes that produce unique experiences for each group. As a final note we provide a caveat. Be prepared for the unexpected. Just when you think that a group will be competitive, they will be cooperative. Just when you think a group will be successful and satisfied because a vision and support were provided by the leader, they will be unhappy. Still, it is our experience that under each unexpected event there is an important aspect of organizational behavior to be explored. It therefore becomes the challenge for the instructor to carefully observe and discover the complexity of the intergroup and interpersonal dynamics that occur in these exercises. 